\title{
THIRD-ORDER NONLINEAR OPTICAL PRPOPERTIES OF POLYDIACETYLENES
}

\author{
Kenji TAKEDA ${ }^{a}$, Tatsuo HASEGAWA ${ }^{b}$, Ken ISHIKAWA ${ }^{c}$ \\ and Takao KODA ${ }^{b}$
}

\author{
a Tsukuba Research Laboratory,Japan Synthetic Rubber Co.Ltd., \\ Tsukuba 305,JAPAN \\ b Department of Applied Physics,Faculty of Engineering,University of Tokyo,Tokyo \\ $113, J A P A N$ \\ c Department of Organic and Polymeric Materials, Faculty of Engineering,Tokyo \\ Institute of Technology,Tokyo 152,Japan
}

Third-order nonlinear optical properties of highly oriented polydiacetylene thin films in blue- and red-form have been studied by means of third harmonic generation (THG) and electro-absorption (EA) measurements. Basing on the respective results of measurements on $\operatorname{Im} \mid \chi^{(3)}(-3 \omega ; \omega$, $\omega, \omega) \mid$ and $\operatorname{Im} \chi^{(3)}(-\omega ; 0,0, \omega)$, the difference of the third-order nonlinear optical susceptibilities of polydiacetylenes in blue- and red-form was explained by the three-level model for quashi-one-dimentional excitons on polyme backbone chains. The important roles of the two-photon resonance effect for ${ }^{1} A_{g}$ exciton state as well as the three-photon resonance effect for the ${ }^{1} B_{\mathfrak{u}}$ exciton state to the optical nonlinealities of polydiacetylenes were demonstrated.

\section{Introduction}

Polydiacetylenes are known as a group of unique conjugative polymers with extended chain structure, being aligned into a definite direction to compose nearly defect-free polymer single crystals. Because of large nonlinear optical susceptibility [1] and short responce time [2], polydiacetylenes have been regarded as one of the most promising and attractive materials, however, the origin and mechanism of their nonlinearities are not fully understood, as yet. It might be partly due to the difficulties of getting high quality samples for the exact measurements and the efforts have been devoted to overcome the problems. In the course of study, we have succeeded in fabricating the highly oriented polydiacetylene thin films in blueand red-form by vacuum deposition process [3] , which made it possible to get more informations and deeper insight about them. In this paper the experimental results on third- 
order nonolinear optical properties of polydiacetylenes are going to be discussed by the threelevel model, emphasizing the contribution of the two-photon resonance effect for the ${ }^{1} \mathrm{~A}_{\mathrm{g}}$ exciton state as well as the three-photon resonance effect for the ${ }^{1} B_{\mathbf{u}}$ exciton state.

\section{Method}

\section{Sample Preparation}

Diacetylene monomers used in the present study are the alkylurethane substituted ones with the molecular formula of $\left.\mathrm{R}-\mathrm{C} \equiv \mathrm{C}-\mathrm{C} \equiv \mathrm{C}-\mathrm{R}\left[\mathrm{R}=\left(\mathrm{CH}_{2}\right)_{\mathrm{m}} \mathrm{OCONHC} \mathrm{n}_{\mathrm{n}} \mathrm{H}_{2 \mathrm{n}+1}\right) ; \mathrm{C}_{\mathrm{m}} \mathrm{UC}_{\mathrm{n}}\right]$, being synthesized by either the reaction between diyne-diol and alkylisocyanate or between diyne-dichlorocarbonate and alkyl-amine [4] . A series of $\mathrm{C}_{4} \mathrm{UC}_{\mathrm{n}}$ were mainly employed by the reason that $\mathrm{C}_{4} \mathrm{UC}_{\mathrm{n}}$ are the appropriate monomers for the thin film fabrication by the vacuum deposition process, because of littl thermal- and fairly large photo-polymerizability, as is shown in Fig.1. Highly oriented poly- $\mathrm{C}_{4} \mathrm{UC}_{\mathrm{n}}$ thin film in blue-form was prepared by two steps vacuum deposition process, details of which are described in the previous papers [3] . The oriented red-form film was obtained by thermochromic conversion of the blue-form film at a given temperature lower than its melting point. The excellent red-form thin film with spectroscopically sharp exciton peak was obtained by thermal annealing of the partially polymerized (up to $50 \%$ ) blue-form film, in order to minimize the conformation disorder effect. The thermochromic phase transition behaviors of poly- $\mathrm{C}_{4} \mathrm{UC}_{4}$ thin films from blue- to red-form are summerized in Fig.2.
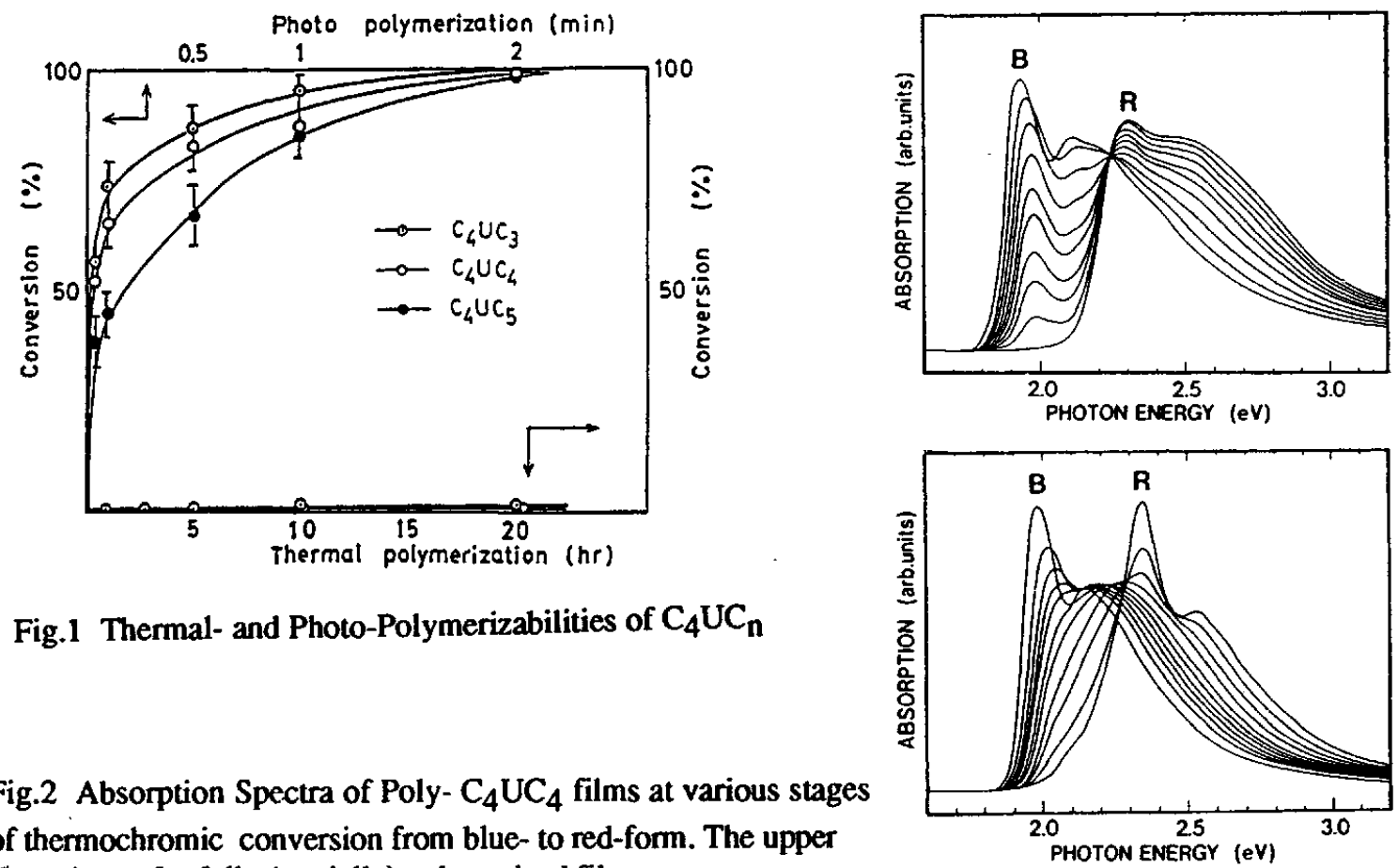

Fig. 2 Absorption Spectra of Poly- $\mathrm{C}_{4} \mathrm{UC}_{4}$ films at various stages
of thermochromic conversion from blue- to red-form. The upper (lower) part for fully (partially) polymerized films 


\section{Third Harmonic Generation (THG) Measurements}

The THG measurements were made on the blue- and red-form films, using pulsed fundamental laser beams with the pulse duration of $5.5 \mathrm{~ns}$ and the power density of 80 $\mathrm{MW} / \mathrm{cm}^{2}$. The fundamental laser beam was obtained in combination with a Q-switched Nd:YAG laser, a dye laser and a difference frequency generator. The exciton resonance enhancement effect was studied by scanning the photon energy of fundamental laser pulse from 0.60 to $0.85 \mathrm{eV}$. The $\left|\chi^{(3)}(-3 \omega ; \omega, \omega, \omega)\right|$ spectra were evaluated by the Maker fringe method, utilizing $1 \mathrm{~mm}$-thick quartz plate as the standard sample. The effects of internal attenuation and reflection of THG lights was taken into account, since the THG radiation is in the strong exciton absoption region.

\section{Electro-Absorption (EA) Measurements}

The EA measurements were made on the same oriented thin films in blue- and red-form as the ones used for the THG measurements. An AC electric field of about $16 \mathrm{kV} / \mathrm{cm}$ with the frequency of $1 \mathrm{kHz}$ was applied parallel to the oriented axis across the evaporated aluminum electrodes deposited on the sample surfaces. (For the perpendicular fields, the EA signals were much smaller than the parallel modulation.) The modulated transmittance signals $(\Delta T)$ were detected at the second harmonic frequency $(2 \mathrm{kHz})$ and the modulated absorption spectra $(\Delta \alpha 1=-\Delta T / T)$ can be obtained, dividing by the tansmittance $(T)$. The modulated dielectric spectra $(\Delta \varepsilon)$ was derived from the Kramers-Kronig transformation of the $\Delta \alpha$ spectra. The imaginary part $\left(\Delta \varepsilon_{2}\right)$ of $\Delta \varepsilon$ is related to the imaginary part of thirdorder nonlinear optical susceptibility $\chi(3)(-\omega ; 0,0, \omega)$ by the relation;

$$
\Delta \varepsilon_{2} / \varepsilon_{0}=3 \operatorname{Im} \chi^{(3)}(-\omega ; 0,0, \omega) \mathrm{E}^{2}
$$

\subsection{Results and Discussion}

The polydiacetylene thin films fabricated by vacuum deposition processin the present study showed a high degree of optical anisotropy with an optical density ratio of about 30 . The THG intensities parallel to the oriented polymer chains were nearly four orders of magunitude larger than those for the parpendicular ones. The experimental $\chi^{(3)}$ values were evaluated from the observed relative THG intensities and the film thickness according to the simplified equation proposed by Kubodera et al [5] .

Fig.3 shows the $\left|\chi^{(3)}(-3 \omega ; \omega, \omega, \omega)\right|$ spectra of thin films both in blue- and redform, in which the prominent three-photon resonance at the respective exciton peaks at 1.95 and $2.35 \mathrm{eV}$ can be clearly observed.

In Fig.4 is plotted the experimental $\operatorname{Im} \chi^{(3)}(-\omega ; 0,0, \omega)$ spectra together with the energy derivatives of $\operatorname{Im} \chi^{(1)}$ spectra represented by the broken curves. 


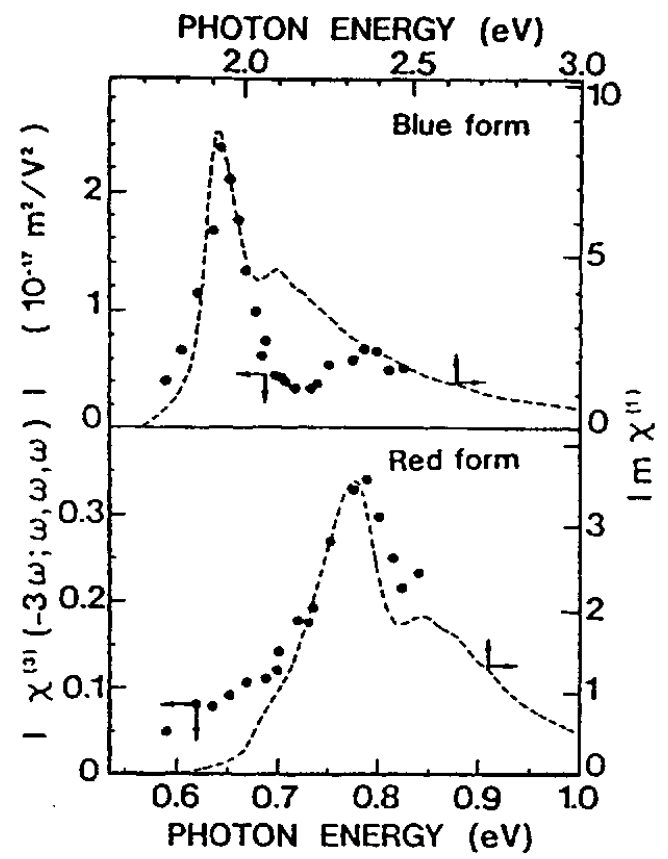

Fig.3 Third-order nonlinear optical susceptibility spectra $\chi^{(3)}(-3 \omega ; \omega, \omega, \omega)$ of blue- and red-form poly- $\mathrm{C}_{4} \mathrm{UC}_{4}$ films in comparison with the exciton absoption spectra $\left(\operatorname{Im} \chi^{(1)}\right)$

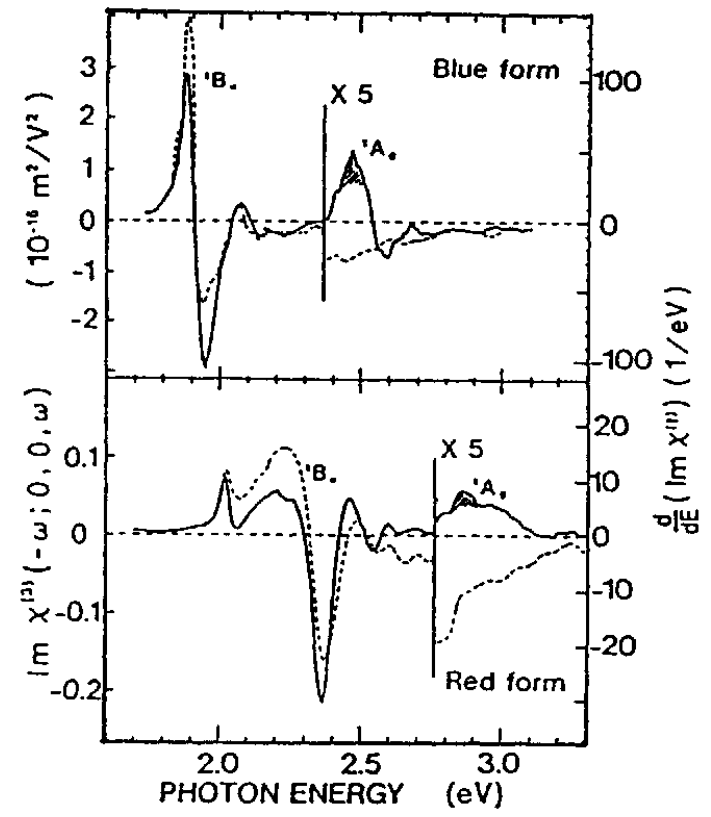

Fig.4 Third-order nonlinear optical susceptibility spectra $\left(\operatorname{Im} \chi^{(3)}(-\omega ; 0,0, \omega) \omega\right)$ of blue- and red-form poly-C ${ }_{4} \mathrm{UC}_{4}$ in comparison with the energy derivative absorption spectra

According to the three-level model for THG and EA processes, $\left|\chi^{(3)}(-3 \omega ; \omega, \omega, \omega)\right|$ and $\operatorname{Im} \chi^{(3)}(-\omega ; 0,0, \omega)$ are given by the following expressions, respectively.

$\left|\chi^{(3)}(-3 \omega ; \omega, \omega, \omega)\right|=\frac{\mathrm{Ne}^{4} \mathrm{~L}_{\mathrm{f}}^{4} \quad \mathrm{f}_{\mathrm{AX}}}{24 \mathrm{~m}^{2} \mathrm{~h}_{0} \omega_{\mathrm{A}}\left(\omega_{\mathrm{X}}-\omega_{\mathrm{A}}\right)\left(\omega_{\mathrm{A}}-\omega\right)\left(\omega_{\mathrm{X}}-2 \omega\right)} \frac{\mathrm{f}_{\mathrm{GA}}}{\left.\sqrt{\left\{\left(\left(\omega_{\mathrm{A}}-3 \omega\right)^{2}+\Gamma_{\mathrm{A}}{ }^{2}\right.\right.}\right\}}$

$\operatorname{Im} \chi^{(3)}(-\omega ; 0,0, \omega)=\frac{\left.N L_{f}^{4}<G|\mu| A><A|\mu| X><X|\mu| A><A|\mu| G\right\rangle}{3 \hbar^{3} \varepsilon_{0}\left(\omega_{A}-\omega-i \Gamma_{A}\right)\left(\omega_{A}-\omega-i \Gamma_{A}\right)\left(\omega_{X}-\omega-i \Gamma_{X}\right)}$

$\cong \frac{1 L_{f}^{2}|<A| \mu|X>| 2}{3} \frac{d}{E_{X}-E_{A}}\left(\operatorname{Im} \chi^{(1)}\right) \quad:$ (near $\left.\omega_{A}\right)$

$\cong \frac{1 L_{\mathrm{f}}^{2}|<\mathrm{A}| \mu|\mathrm{X}>|^{2} N L_{\mathrm{f}}^{2}|<\mathrm{G}| \mu|\mathrm{A}>|^{2} \Gamma_{\mathrm{X}}}{3\left(\mathrm{E}_{\mathrm{X}}-\mathrm{E}_{\mathrm{A}}\right)^{2}}:$ (near $\omega_{\mathrm{X}}$ ) 
The notations are $; L_{f}$ (local field factor), $N$ (unit cell density), $\langle i|\mu| j\rangle$ (dipole matrix element), $f_{i j}$ (oscillator strength), and $\Gamma_{\mathrm{i}}$ (damping constant). The experimental results in Fig. 3 and Fig. 4 were applied to the equations above, and the relative ratio of the nonlinear optical response of the blue- and red-form films was estimated to be about 2.9 . In the Im $\chi^{(3)}(-\omega ; 0,0, \omega)$ spectra, the major structures observed near the respective exciton peaks can be attributed to the effect of field-induced shift of energies, which is represented by eq.(4). The spectral shapes are well fitting to the energy derivative curves of $\operatorname{Im} \chi^{(1)}$ spectra shown by the broken lines. The structures in the higher energy region (indicated by hatched area) are attributed to the optical forbidden ${ }^{1} \mathrm{~A}_{\mathrm{g}}$ exciton, which is activated by the electric field according to eq.(5). It is concluded that the forbidden (two photon allowed) ${ }^{1} \mathrm{~A}_{\mathrm{g}}$ excitons are located about $0.5 \mathrm{eV}$ higher than the lowest allowed ${ }^{1} \mathrm{~B}_{\mathrm{u}}$ excitons in both form polydiacetylene films. From the analysis of $\operatorname{Im} \chi^{(3)}(-\omega ; 0,0, \omega)$ spectra, the value of $f_{A X}$ can be estimated, which are summerized in Fig.5, together with other parameters estimated from the Im $\chi^{(1)}$ spectra. Those results are consistent with the experimental $\left|\chi^{(3)}(-3 \omega ; \omega, \omega, \omega)\right|$ spectra shown in Fig.3. The larger nonlinear optical response of the blue-form than the redform is predominantly due to the contribution of $f_{\mathrm{AX}}$ in eq.(2), which is about 3.2 times larger for the blue-form than the red-form [6] .

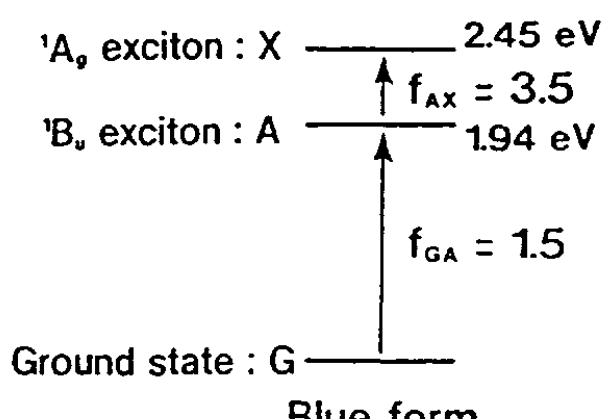

Blue form

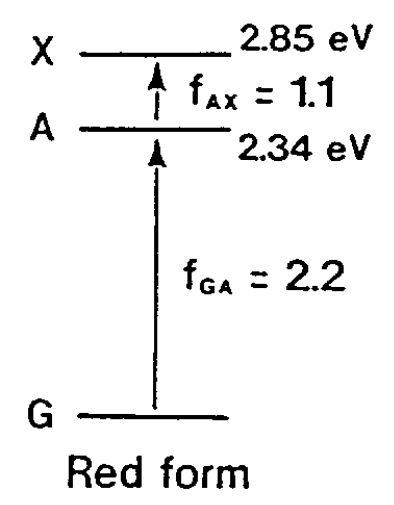

Fig.5 Three-level models for blue- and red-form poly- $\mathrm{C}_{4} \mathrm{UC}_{4}$

From our recent studies on the phase and modulus of complex $\chi^{(3)}(-3 \omega ; \omega, \omega, \omega)$ values of poly- $\mathrm{C}_{4} \mathrm{UC}_{4}$ in blue-form, the two-photon resonance effect for the ${ }^{1} \mathrm{Ag}_{\mathrm{g}}$ exciton state as well as the three-photon resonance effect for the ${ }^{1} B_{\mathbf{u}}$ exciton state have been experimentally confirmed [7] . The outline of the results are as follows:

(1) The phase spectra show a step-wise feature at about $0.6 \mathrm{eV}$, where the phase increases sharply from $0^{\circ}$. Note that this step in the phase spectra coincides with a sharp peak in the modulus spectra. At the energy of the modulus peak at about $0.65 \mathrm{eV}$, the phase reaches $90^{\circ}$ and increases further toward $180^{\circ}$ on the higher energy side. This gives evident support for the interpretation that these features are due to the three-photon resonance effect for the lowest ${ }^{1} \mathrm{~B}_{\mathrm{u}}$ exciton at $1.95 \mathrm{eV}$. 
(2) The phase remains nearly constant $\left(=170^{\circ}\right)$ up to about $0.81 \mathrm{eV}$, which contradicts an interpretation that the second peak in the modulus spectra is due to the two-photon resonance with a lower-lying forbidden exciton [8]. We conclude that this feature is attributable to the three-photon resonance to a higher-lying ${ }^{1} B_{\mathbf{u}}$ exciton or to the band edge.

(3) The phase spectra seem to show a second step, from $\phi=170^{\circ}$ to $280^{\circ}$, at an energy between 0.81 and $1.15 \mathrm{eV}$. This step should be attributed to the two-photon resonance effect by the forbidden ${ }^{1} \mathrm{~A}_{\mathrm{g}}$ exciton and the result is consistent with the three-level model based on the electro-absorption spectra. According to the model, the ${ }^{1} \mathrm{~A}_{\mathrm{g}}$ exciton is located about $2.45 \mathrm{eV}$, which predicts a modulus peak and a phase step in the $\chi^{(3)}$ spectra at about $1.22 \mathrm{eV}$.

\section{Conclusion}

By the appropriate molecular design and screaning of diacetylene compounds, highly oriented polydiacetylene thin films in blue- and red-form could be prepared from alkylurethane subsituted monomers by vacuum deposition process. The difference of opticalnonlinealities of blue- and red-form was clearly explained by the three level model, in which the roles of twophoton resonance effect for ${ }^{1} A_{g}$ state as well as the three-photon resonance effect for the ${ }^{1} B_{u}$ exciton state are demonstrated.

\section{Acknowledgements}

This work was supported in part by a Research Grant-in-Aid from the Ministry of Education, Culture and Science in Japan, the Nissan Science Foundation and of the NEDO Foundation, Japan.

\section{References}

1. C.Sauteret,J.P.Hermann,R.Frey,F.Prandere,J.Ducuing,R.H.Baughman and R.R.Chance, Phys.Rev.Letters.36(1976)956

2. For a review, see Nonlinear Optical Properties of Organic Molecules and Crystals, edited by D.S.Chemla and J.Zyss(Academic, Orlando,1987),Vol.2, Chap.III-1,2,3

3. T.Kanetake,K.Ishikawa,T.Hasegawa,T.Koda,K.Takeda,M.Hasegawa,K.Kubodera and H.Kobayashi,Appl.Phys.Lett.54(1989)2287.

4. K.Takeda,T.Koda,S.Koshihara and Y.Tokura,Synth.Metals,41-43(1991)231

5. K.Kubodera and T.Kaino, in : Nonlinear optics in organics and semiconductors, ed. T.Kobayashi Springer,Berlin,1989)

6. T.Hasegawa,K.Ishikawa,T.Koda,K.Takeda,H.Kobayashi and K.Kubodera, Synth.Metals,41-43(1991)3151

7. T.Hasegawa,K.Ishikawa,T.Koda,K.Takeda,H.Kobayashi and K.Kubodera, Synth.Metals,49-50(1992)123

8. P.Chollet,F.Kajar and J.Messier,Synth.Metals,18(1987)459 